Europhys. Lett., 74 (4), pp. 588-594 (2006)

DOI: $10.1209 /$ epl/i2005-10565-4

\title{
Stationary Hamiltonian transport with dc bias
}

\author{
S. Denisov ${ }^{1}$, S. FlaCH ${ }^{1}$ and P. HÄNGGi ${ }^{1,2}$ \\ 1 Max-Planck Institut für Physik Komplexer Systeme - Nöthnitzer Str. 38 \\ D-01187 Dresden, Germany \\ 2 Universität Augsburg, Institut für Physik - Universitätsstrasse 1 \\ D-86135 Augsburg, Germany
}

received 16 December 2005; accepted in final form 15 March 2006

published online 12 April 2006

PACS. 05.45. Ac - Low-dimensional chaos.

PACS. 05.60.-k - Transport processes.

\begin{abstract}
The dynamics of a particle in a symmetric periodic potential under the influence of a time-periodic field is characterized by a mixed phase space with regular and chaotic components. An additional external dc bias transforms the chaotic manifold into a domain with unbounded acceleration. We study the stationary transport which originates from the persisting invariant manifolds (regular islands, periodic orbits, and cantori) that are initially embedded in the chaotic manifold. We prove persistence and emergence of transporting islands. The transient dynamics of the accelerated domain separates fast chaotic motion from ballistic type trajectories which stick to the vicinity of the invariant submanifold. Experimental studies with cold atoms in laser-induced optical lattices are ideally suited for testing and observing our findings.
\end{abstract}

The main mechanisms governing Hamiltonian transport are strongly related to a mixed phase space with coexisting regular and chaotic regions [1,2]. Modern experimental research using manipulations with cold atoms ensembles in optical potentials provides a testing ground to explore this issue [3]. The driven pendulum, a paradigmatic system for the study of dynamical chaos [2], is realized using a periodically modulated optical standing wave, with the possibility to control the strength of dissipation down to arbitrarily small values [3]. Mixed phase space structures have been resolved already with cold atom experiments [4].

Mixed phase space structures are also at the heart of the recently discussed directed chaotic transport in driven Hamiltonian systems, so-called Hamiltonian ratchets [5-7]. Recent experiments [8] study the crossover from dissipative to Hamiltonian ratchets, confirming these theoretical predictions.

What will happen to a driven Hamiltonian system when exposed to an additional dc bias? Contrary to the common expectation that the trajectories acquire unbounded acceleration, it has very recently been found in biased Hamiltonian ratchet systems that this does not hold true for all trajectories [9]. Again, the main reason for that finding is a mixed phase space structure of the unbiased system [9].

In this letter we systematically explore the route of obtaining a stationary Hamiltonian transport in the presence of a dc bias. While the chaotic phase space regions transform into accelerated evolution for any arbitrary small value of the dc bias, regular transporting 
submanifolds (unstable periodic orbits, regular islands and cantori with nonzero average velocities), initially embedded in the chaotic region, persist under finite dc bias. Even more, some transporting unstable periodic orbits at zero dc bias lead to the appearance of stable transporting islands for some finite nonzero values of the dc bias. The presence of cantori [10] and the corresponding sticking of chaotic trajectories in the vicinity of regular manifolds has a strong impact on the transient dynamics of chaotic phase space parts which are close to the above-mentioned regular manifolds.

We consider the canonical Hamiltonian model of a particle moving in a symmetric (i.e. non-ratchet-type) one-dimensional space-periodic potential $U(X)=\frac{1}{2 \pi} \cos (2 \pi x)$ under the influence of a time-periodic space-homogenous external field $E(t)=E_{a c} \sin (\omega t)$ [2], to which we add an external dc bias $E_{b}$ :

$$
\dot{x}=p, \quad \dot{p}=\sin (2 \pi x)+E_{a c} \sin (\omega t)-E_{b} .
$$

Due to time and space periodicity of the system we can map the original three-dimensional phase space $(x, p, t)$ onto a two-dimensional cylinder, $\mathrm{T}^{2}=(x \bmod 1, p)$, by using the stroboscopic Poincaré section after each period $T=2 \pi / \omega$, cf. fig. 1 (a).

Let us start out with the case of zero dc bias $E_{b}=0$. Then, the phase space of the system is characterized by the presence of a stochastic layer which originates from the destroyed separatrix of the undriven case $E_{a c}=0$ [2]. The chaotic layer is confined by transporting KAM-tori, which originate from perturbed trajectories of particles with large kinetic energies. These tori are noncontractible (since they cannot be continuously contracted to a point on $\mathrm{T}^{2}$ ), and separate the cylinder.

The stochastic layer is not uniform and contains different regular invariant manifolds. There is an infinite number of periodic orbits (POs) embedded in the layer. Each PO, $\hat{\boldsymbol{X}}_{T_{p}}(t)=$ $\left\{x_{T_{p}}(t), p_{T_{p}}(t)\right\}$, is characterized by the period $T_{p}=k T, k=1,2, \ldots$, an integer shifting distance $L$, and a winding number $v=L / T_{p}$ :

$$
x_{T_{p}}\left(t+T_{p}\right)=x_{T_{p}}(t)+L, \quad p_{T_{p}}\left(t+T_{p}\right)=p_{T_{p}}(t) .
$$

If $v \neq 0$ then that PO is transporting. POs can be linearly stable or unstable [11]. Towards this goal we linearize the phase space flow around a PO $\hat{\boldsymbol{X}}_{T_{p}}(t)$, and map it onto itself by integrating over one period $T_{p}$. The resulting $2 \times 2$ symplectic Floquet matrix $\boldsymbol{M}$ has eigenvalues (Floquet multipliers) $\lambda_{1}$ and $\lambda_{2}$ with $\lambda_{1} \lambda_{2}=1$ [11]. For a stable PO, both multipliers are located on the unit circle, while for an unstable PO, both multipliers are located either both on the negative or positive real axis.

Stable POs are always enclosed by regular islands, which are filled by contractible tori, cf. fig. 1(a). If a stable PO is transporting then the corresponding island is also transporting with the same winding number $v$. In addition, within the chaotic sea there is an infinite number of unstable POs with different periods and winding numbers. They are unstable with respect to small perturbations and are not isolated from the chaotic layer. Finally, cantori [10] exist which generate semipenetrable barriers in phase space.

Equation (1) is invariant under time reversal

$$
S: t \rightarrow-t+T / 2, \quad x \rightarrow x, \quad p \rightarrow-p,
$$

which changes the sign of the current $J=v$. Thus, transporting invariant POs and islands appear as symmetry-related pairs in phase space with opposite winding numbers. Consequently, no overall net transport occurs in this driven but unbiased situation $[5-7,9]$.

Let us focus now on the case $E_{b} \neq 0$. In fig. 1(b) we plot the escape time $T_{\text {esc }}$ as a function of initial conditions $\left\{x_{0}, p_{0}\right\}$ for nonzero $E_{b}$. The window $\left\{x_{0}, p_{0}\right\}$ coincides with fig. $1(\mathrm{a})$. The 

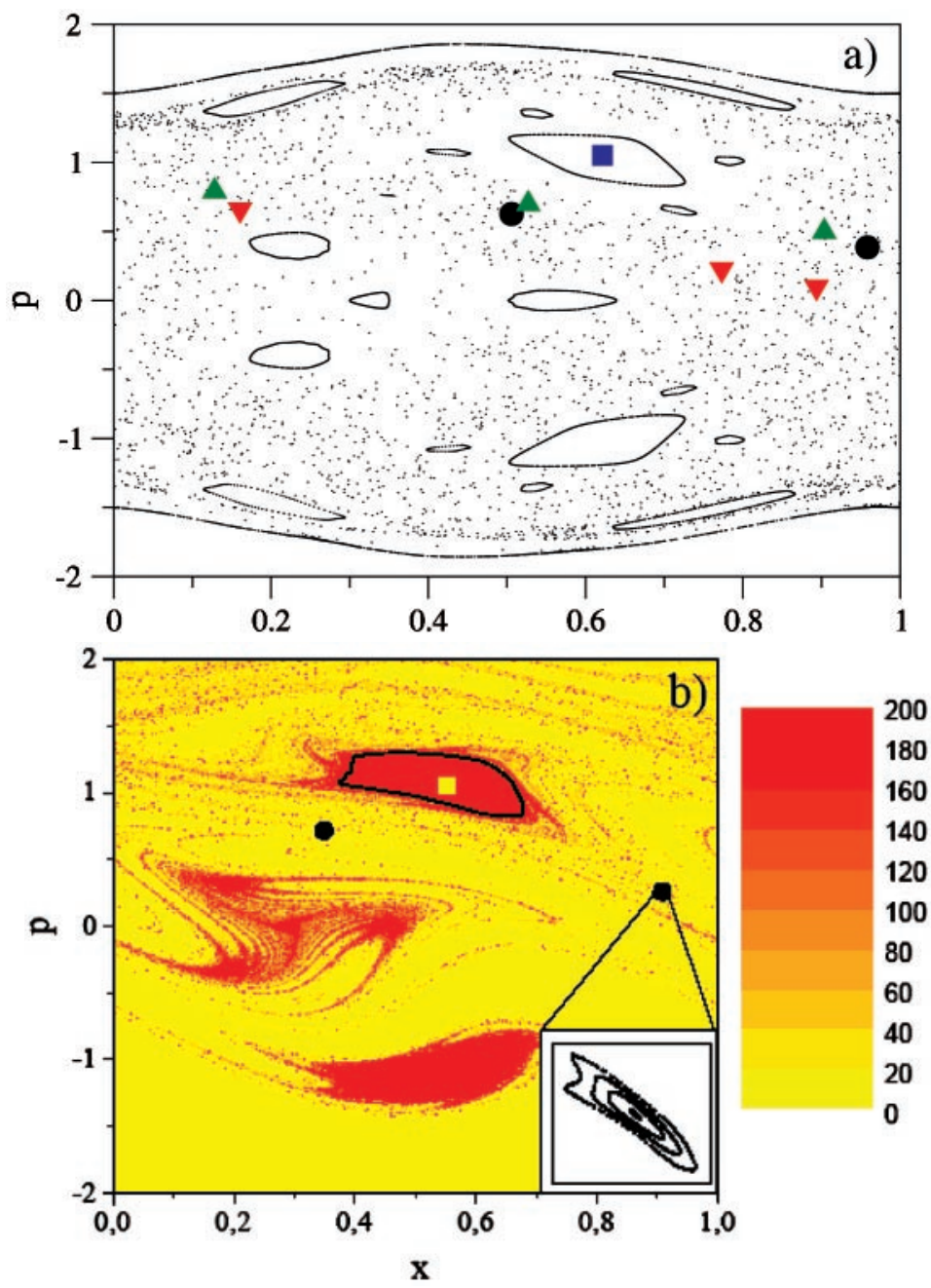

Fig. 1 - (a) Poincaré section for the system equation (1), $\omega=2 \pi, T=1, E_{a c}=5.8$, for (a) $E_{b}=0$ and (b) $E_{b}=0.183$. Several POs are shown: one stable $(k=1, L=1)$ (squares), and several unstable POs ( $k=2, L=1)$ (circles), ( $k=3, L=2)$ (upward triangles), and ( $k=3, L=1)$ (downward triangles). The island group near $p=0$ has zero winding number $v=0$. (b) Time $T_{\text {esc }}$ to be accelerated until $p\left(T_{\text {esc }}\right)=-10$ as a function of the initial conditions in phase space. Trajectories from the islands are not accelerated at all. Inset in (b): Poincaré section zoom of phase space structure showing the transformation of an unstable PO into a stable PO with a surrounding invariant regular island.

escape time is reached by a trajectory if $p\left(T_{\text {esc }}\right)=-10$, and we integrate up to $t=200$. While most of the chaotic layer in fig. 1(a) is quickly accelerated, regular islands persist and their trajectories are in fact not accelerated at all. Moreover we observe phase space regions with delayed acceleration. These trajectories stick for a large time to the persisting regular islands.

Noncontractible KAM-tori do not survive in the biased Hamiltonian system. If there exists at least one accelerating trajectory, then KAM-tori do not persist [9,12]. Such accelerating trajectories always exist for $E_{b} \neq 0$, both with $E_{a c}=0$ and $E_{a c} \neq 0$.

Let us give analytical proof that POs at $E_{b}=0$ persist for nonzero $E_{b}$. Denote a solution of (1) with initial conditions $\boldsymbol{X}_{0}=\left\{x_{0}, p_{0}\right\}$ at $t=0$ by $\hat{\boldsymbol{X}}\left(t, \boldsymbol{X}_{0}, E_{b}\right)=$ 


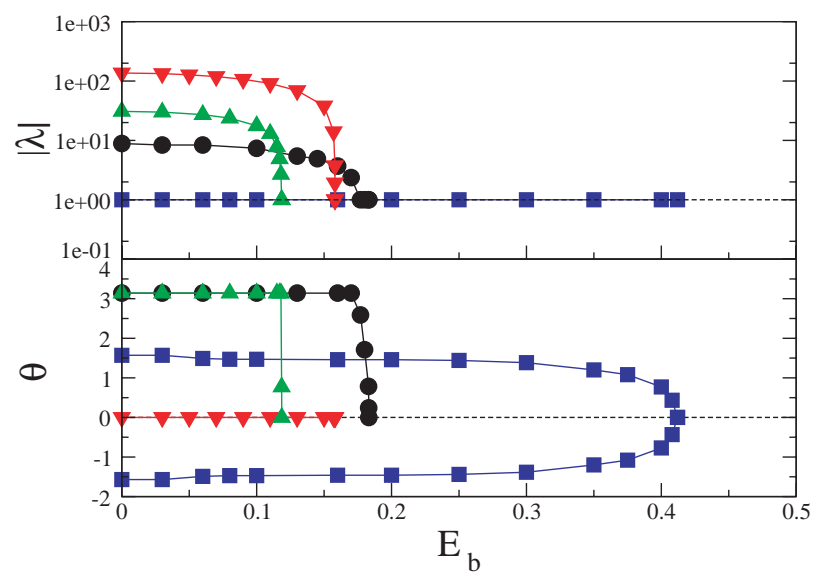

Fig. 2 - Variation of Floquet multipliers, $\lambda_{j}=\left|\lambda_{j}\right| e^{i \theta_{j}}$, of POs vs. bias $E_{b}$ for eq. (1) with parameters and symbols as in fig. 1 . We show both multipliers for stable PO (squares) and $\left|\lambda_{1}\right|>1$ for unstable POs.

$\left\{x\left(t, x_{0}, p_{0}, E_{b}\right), p\left(t, x_{0}, p_{0}, E_{b}\right)\right\}$. The trajectory $\hat{\boldsymbol{X}}\left(t, \boldsymbol{X}_{0}, E_{b}\right)$ is $T_{p}$-periodic if and only if the vector function

$$
\boldsymbol{F}\left(\boldsymbol{X}_{0}, E_{b}\right)=\left(\begin{array}{c}
x\left(T_{p}, x_{0}, p_{0}, E_{b}\right)-x_{0}-L \\
p\left(T_{p}, x_{0}, p_{0}, E_{b}\right)-p_{0}
\end{array}\right)=\mathbf{0}
$$

For $\boldsymbol{X}_{0}=\left\{x_{T_{p}}(0), p_{T_{p}}(0)\right\} \hat{\boldsymbol{X}}\left(t, \boldsymbol{X}_{0}, 0\right) \equiv \hat{\boldsymbol{X}}_{T_{p}}(t)$. Then eq. (4) is satisfied by $\hat{\boldsymbol{X}}_{T_{p}}(0)$ at $E_{b}=0$. By the Implicit Function Theorem [13], if the Jacobian of the vector function $\boldsymbol{F}\left(\boldsymbol{X}_{0}, E_{b}\right)$ with respect to $\boldsymbol{X}_{0}$ is nonzero at $\hat{\boldsymbol{X}}_{T_{p}}(0), E_{b}=0$, then the solution $\hat{\boldsymbol{X}}_{T_{p}}(0)$ can be continued to $E_{b} \neq 0, \boldsymbol{X}_{0}\left(E_{b}\right)=\left\{x_{0}\left(E_{b}\right), p_{0}\left(E_{b}\right)\right\}$ which corresponds to a $T_{p}$-periodic orbit of the perturbed system. The Jacobian of the function (4) at $\hat{\boldsymbol{X}}_{T_{p}}(0)$ is $\operatorname{det} \partial \boldsymbol{F} / \partial \boldsymbol{X}=\operatorname{det}[\boldsymbol{M}-\boldsymbol{I}]$, where $\boldsymbol{I}$ is the unity matrix. The Jacobian is nonzero if $\lambda_{1,2} \neq 1$ for $\boldsymbol{M}$. Thus, all POs of the unperturbed system with $\lambda_{1,2} \neq 1$ persist for nonzero dc bias. Such POs can be continued up to a value of $E_{b}$, where their Floquet multipliers collide at +1 . Linearly stable POs in Hamiltonian systems are always enclosed by tori, which form a regular island. Thus, the transporting contractible islands also persist for nonzero dc bias. Note that the symmetry (3) also persists for nonzero $E_{b}$. Consequently, any invariant transporting manifolds which persist for nonzero $E_{b}$ come in pairs and stationary currents occur in both directions [5].

We expect that for large enough dc bias $E_{b} \gg 1$, a transporting PO will disappear. This occurs when its Floquet multipliers collide at $+1, \lambda_{1}\left(E_{b}^{c}\right)=\lambda_{2}\left(E_{b}^{c}\right)=1$. This is a saddlecenter bifurcation [14], when two POs of the same period coalesce, one of them being initially stable and the other one unstable. Since stable POs are enclosed by regular islands, this can happen only when the corresponding island surrounding the stable PO shrinks to zero.

In fig. 1(a) we depict the phase space structure of eq. (1) for $\omega=2 \pi, E_{a c}=5.8$ and $E_{b}=0$. Using a Newton method [14], we find stable and unstable POs (various symbols in fig. 1), and continue them to nonzero values of $E_{b}$. Stable POs exist up to $E_{b} \approx 0.412$. The evolution of the corresponding Floquet multipliers $\lambda=|\lambda| \exp [i \theta]$ is depicted in fig. 2.

Transporting unstable POs become more stable with increasing dc bias, since they have to acquire $\lambda_{1,2}=1$ at a critical dc bias value before disappearing. Moreover, unstable POs with negative real Floquet multipliers have to become stable POs before a critical bifurcation 
occurs, since the only way to move the multipliers from the negative real axis to +1 is to transport them towards -1 , and subsequently to shift them along the unit circle. During that last stage an originally unstable PO transforms into a stable PO, and a new transporting regular island emerges in phase space. Thus, along with an overall shrinking of the size of transporting regular islands with increasing $E_{b}$, new islands are born as well. In the inset in fig. 1(b) a zoom of the Poincaré section exhibits such a case. For $E_{b}=0$ we found an unstable PO with $T_{p}=2$ and winding number $v=1 / 2$ and $\lambda_{1} \approx-8.365, \lambda_{2}=1 / \lambda_{1} \approx-0.119$. This orbit becomes stable at $E_{b} \approx 0.178$ (when the multipliers collide at -1 and enter the unit circle) and disappears at $E_{b} \approx 0.185$, when $\lambda_{1}=\lambda_{2}=1$. In between these two bias values the now stable PO is surrounded by a regular island of finite size.

For any $\mathrm{PO}$ the momentum $p(t)$ is a periodic function and can be represented in the form $p(t)=v+p^{0}(t)$, such that $p^{0}\left(t+T_{p}\right)=p^{0}(t),\left\langle p^{0}(t)\right\rangle_{T_{p}}=0$. Then we obtain the energy balance equation:

$$
v E_{b}=\left\langle p^{0}(t) E_{a c}(t)\right\rangle_{T_{p}} .
$$

An external ac field mimics an additional effective thermostat: it pumps energy into the system in order to compensate the work against bias (for POs with positive $v$ ) and acts as an energy sink to compensate the acceleration in the case of current along a bias (for POs with negative $v$ ). Notably in our case the generating equations are Hamiltonian and dissipationless.

The originally chaotic trajectories will be accelerated without bound for nonzero $E_{b}$. Nevertheless, the mixed phase space structure for $E_{b}=0$ leads to a strongly nonuniform acceleration in time for trajectories with different initial conditions. That can be already observed in

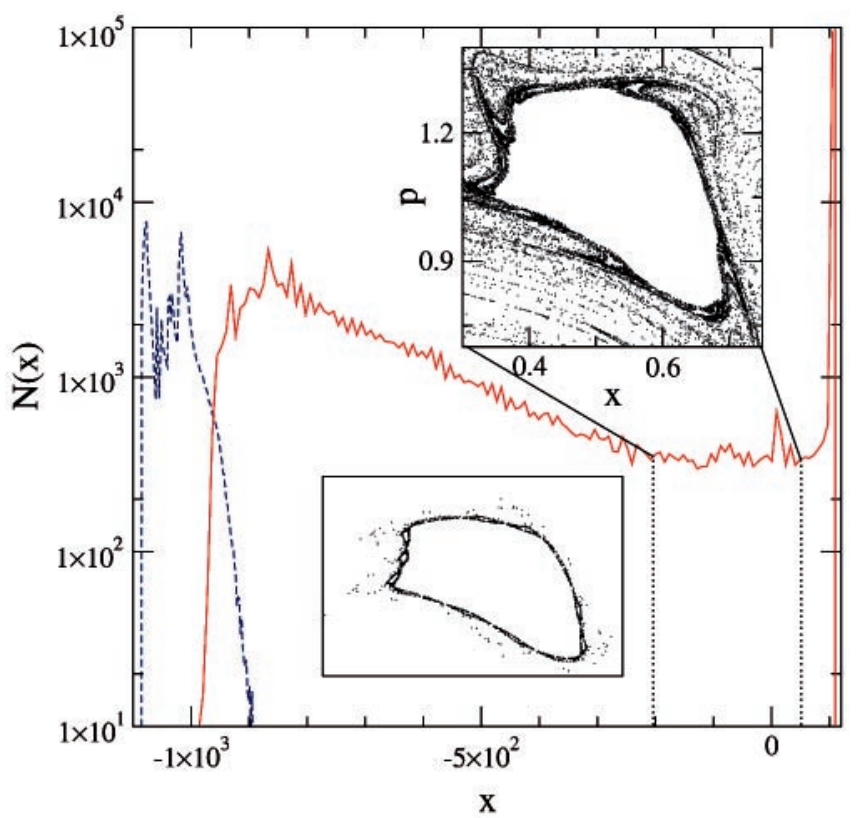

Fig. 3 - Solid line: spatial distribution $N(x)$ after $t=100$ for $\omega=2 \pi, E_{a c}=5.8$ and $E_{b}=0.183$ and when $2.5 \cdot 10^{4}$ trajectories started out from the uniform distribution in the rectangle $(x \in[0.3,0.75], p \in$ $[0.7,1.4])$, which enclose the transporting island with $v=1$. The upper inset shows initial conditions which correspond to the tail of $N(x), x \in[-200,50]$. The lower inset depicts the Poincaré section of a single trajectory that started out in the "sticky" vicinity of the island. Dashed line: distribution $N(x)$ with initial conditions shifted by $\Delta p_{0}=-2.2$ and additional shift in $x$ (see text). 
fig. 1(b). Indeed, for $E_{b}=0$ chaotic trajectories in the vicinity of regular islands may stick to the island boundary for long times and produce a unidirectional flight with the velocity $v$ due to the stickiness effect $[2,15]$. The origin of such a behavior is the presence of partial barriers in phase space formed by cantori [10]. This feature is also observed on finite times for nonzero $E_{b}$. Trajectories may stick for long times to regular island boundaries without acceleration (see the Poincaré section in the lower inset in fig. 3). Only after some rather large escape times $T_{\text {esc }}$ will such a trajectory eventually leave the island vicinity and suddenly accelerate. In fig. 3 (solid line) we compute the distribution $N(x)$ of displacements of trajectories with initial conditions uniformly covering a phase space part which includes a transporting island. We observe that the transient time $T_{\text {esc }}$ can be extremely long for initial conditions in the island's vicinities (see also fig. 1(b)). In fig. 3 the right peak at $x=100$ corresponds to initial conditions inside the island (no acceleration for all times). The left peak at $x=-900$ corresponds to initial conditions that are accelerated right from the beginning. In the absence of trajectories which stick to regular islands with a broad distribution of escape times, the two peaks would be separated by a depleted region, i.e. a gap. Instead we observe a smeared out distribution which corresponds to trajectories with long transient sticking to the island boundary. Indeed, initial conditions which correspond to the gap region stick to the island (upper inset in fig. 3). A shift of the initial conditions by $\Delta p_{0}=-2.2$ down into the homogenous part of the phase space in fig. 1(b) yields a distribution $N(x)$ which contains a single peak due to immediate acceleration (fig. 3, dashed line). Note that we shifted $N(x)$ here by $\Delta x_{0}=220$ which would occurs for particles with $V(x)=0$ and $E_{a c}=0$ if the initial momentum is shifted by $\Delta p_{0}=-2.2$.

In conclusion, we study stationary Hamiltonian transport which is sustained by a nonzero dc bias. The dc bias provides a unique possibility to separate the different parts of a mixed phase space, since it accelerates the chaotic part away and leaves the embedded regular invariant manifolds basically untouched. Moreover, even within the accelerated part, proper distribution functions after finite acceleration times reveal the intriguing properties of surviving cantori [10] and sticking [15]. We give a proof for the persistence of periodic orbits and thus for regular islands. We found evidence for the persistence of cantori as well. Our results are not limited to the case in eq. (1) and are valid as well for the general case of a potential $U(t, x)=U(t+T, x)=U(t, x+L)$. The presence of weak dissipation will not change the situation drastically. Since it can be treated as a smooth perturbation, POs will persist for nonzero damping. Stable POs become attractors (limit cycles), and unstable POs saddles [11]. A side result is then that an infinite number of stable POs for zero damping will lead to an infinite number of attractors for infinitesimally weak damping [16].

A possible testing ground for our results are cold atoms in a standing wave potential [3], where an additional dc bias can be realized either by a gravitational force [17] or through acceleration of an optical lattice [18]. In such a case, all of the above effects could be probed. The persistence of transporting regular islands and POs can be used for the preparation of monochromatic matter waves.

\section{REFERENCES}

[1] Mackay R. S. and Meiss J. D. (Editors), Hamiltonian Dynamical Systems (Adam Hilger, Bristol) 1987.

[2] Zaslavsky G. M., Physics of Chaos in Hamiltonian Systems (Imperial College Press, London) 1998.

[3] Steck D. A., Oskay W. H. and Raizen M. G., Science, 293 (2001) 274; Hensinger W. K. et al., Nature, 412 (2001) 52; Schiavoni M., Sanchez-Palencia L., Renzoni F. and Grynberg G., Phys. Rev. Lett., 90 (2003) 094101. 
[4] Hensinger W. K. et al., Phys. Rev. A, 64 (2001) 033407; Kaplan A., Friedman N., AnDersen M. and Davidson N., Phys. Rev. Lett., 87 (2003) 274101.

[5] Flach S., Yevtushenko O. and Zolotaryuk Y., Phys. Rev. Lett., 84 (2000) 2358.

[6] Goychuk I. and Hänggi P., Lect. Notes Phys., 557 (2000) 7; J. Phys. Chem. B, 105 (2001) 6642.

[7] Schanz H., Отto M.-F., Ketzmerick R. and Dittrich T., Phys. Rev. Lett., 87 (2001) 070601.

[8] Gommers R., Bergamini S. and Renzoni F., Phys. Rev. Lett., 95 (2005) 073003.

[9] Schanz H., Dittrich T. and Ketzmerick R., Phys. Rev. E, 71 (2005) 026228.

[10] Mackay R. S., Meiss J. D. and Percival I. C., Physica D, 13 (1984) 5; Meiss J. D., Rev. Mod. Phys., 64 (1992) 795.

[11] Gutzwiller M. C., Chaos in Classical and Quantum Mechanics (Springer, New York) 1990.

[12] Easton R. W., Meiss J. D. and Roberts G., Physica D, 54 (2001) 201.

[13] Schwartz J. T., Nonlinear Functional Analysis (New York Univ. Lect. Notes, New York) 1964.

[14] Wiggins S., Global Bifurcation and Chaos: Analytical Methods (Springer-Verlag, Heidelberg) 1988.

[15] Geisel T., Nierwetberg J. and Zacherl A., Phys. Rev. Lett., 59 (1987) 2503; Shlesinger M. F., Zaslawsky G. M. and Klafter J., Nature, 363 (1993) 31; Denisov S., Klafter J. and Urbakh M., Phys. Rev. E, 66 (2002) 046217; Altmann E. G., Motter A. E. and Kantz H., Chaos, 15 (2005) 033105.

[16] Feudel U., Grebogi C., Hunt B. R. and Yorke J. A., Phys. Rev. E, 54 (1996) 71.

[17] Anderson B. P. and Kasevich M. A., Science, 282 (1998) 1686.

[18] Wilkinson S. R. et al., Phys. Rev. Lett., 76 (1996) 4512. 